\title{
Unique combination and in silico modeling of biallelic POLR3A variants as a cause of Wiedemann-Rautenstrauch syndrome
}

\author{
Sehime Gulsun Temel $\mathbb{1}^{1,2,3} \cdot$ Mahmut Cerkez Ergoren $\mathbb{1}^{4,5} \cdot$ Elena Manara $\mathbb{D}^{6}$ - Stefano Paolacci $\mathbb{D}^{6}$.

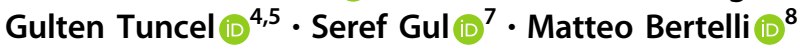

Received: 28 November 2019 / Revised: 19 May 2020 / Accepted: 2 June 2020 / Published online: 18 June 2020

(c) The Author(s), under exclusive licence to European Society of Human Genetics 2020

\begin{abstract}
Neonatal progeroid syndrome or Wiedemann-Rautenstrauch syndrome (WRS; MIM 264090) is a rare genetic disorder that has clinical symptoms including premature aging, lipodystrophy, and variable mental impairment. Until recently genetic background of the disease was unclear. However, recent studies have indicated that WRS patients have compound heterozygote variations in the POLR3A (RNA polymerase III subunit 3A; MIM 614258) gene that might be responsible for the disease phenotype. In this study we report a WRS patient that has compound heterozygote variations in the POLR3A gene. One of the reported variations in our patient, c.3568C $>\mathrm{T}$, p.(Gln1190Ter), is a novel variation that was not reported before. The other variant, c.3337-11T>C, was previously shown in WRS patients in trans with other variations.
\end{abstract}

\section{Introduction}

Neonatal progeroid syndrome, also known as the Wiedemann-Rautenstrauch syndrome (WRS; MIM 264090), is a rare and clinically heterogeneous disorder that

These authors contributed equally: Sehime Gulsun Temel, Mahmut Cerkez Ergoren

Supplementary information The online version of this article (https:// doi.org/10.1038/s41431-020-0673-1) contains supplementary material, which is available to authorized users.

Sehime Gulsun Temel

sehime@uludag.edu.tr

1 Bursa Uludag University, Faculty of Medicine, Department of Medical Genetics, Bursa, Turkey

2 Bursa Uludag University, Faculty of Medicine, Department of Histology and Embryology, Bursa, Turkey

3 Bursa Uludag University, Institute of Health Sciences, Department of Translational Medicine, Bursa, Turkey

4 Near East University, Faculty of Medicine, Department of Medical Biology, 99138 Nicosia, Cyprus

5 Near East University, DESAM Insitute, 99138 Nicosia, Cyprus

6 MAGI Euregio, Bolzano, Italy

7 Koc University, Faculty of Engineering, Department of Chemical and Biological Engineering, Istanbul, Turkey

8 MAGI's LAB S.r.l., Rovereto, Italy is likely to be inherited in an autosomal recessive manner [1]. WRS is characterized by features of premature human aging and showing lipodystrophy as particular local fatty tissue accumulations, craniofacial disproportion (a triangular shape), neonatal dentition, subcutaneous tissue atrophy, hyperinsulinism, sparse scalp hair, and also variable mental impairment [2]. Average life expectancy of WRS patients was around 7 months but there are reports presenting survival into $20 \mathrm{~s}[3,4]$.

RNA polymerase III is a DNA-directed RNA polymerase and it is the largest subunit encoded by POLR3A (RNA polymerase III subunit 3A; MIM 614258). This polymerase transcribes genes coding small RNAs such as ribosomal 5S RNA, tRNA, U6 small nuclear RNA, and mitochondrial RNA-processing RNA. Previously, variations within the POLR3A gene have been associated with two forms of hypomyelinating leukodystrophy with or without oligodontia and/or hypogonadotropic hypogonadism (MIM 607694 and MIM 614381, respectively) [5]. This syndrome is also known as hypomyelination, hypodontia, and hypogonadotropic hypogonadasim $(4 \mathrm{H}$ syndrome or $4 \mathrm{H}$ leukodystrophy) [6]. Variations were identified as homozygous or in compound heterozygous state and no individual had two truncating variations [5]. The underlying genetic cause of the WRS disease was unclear until recent studies indicating that WRS patients have compound heterozygous variants in POLR3A gene that might be responsible for the disease phenotype [4, 7-9]. Jay et al. had identified compound 
Fig. 1 Phenotypic view of our patient. Black arrows indicate local lipoatrophies.

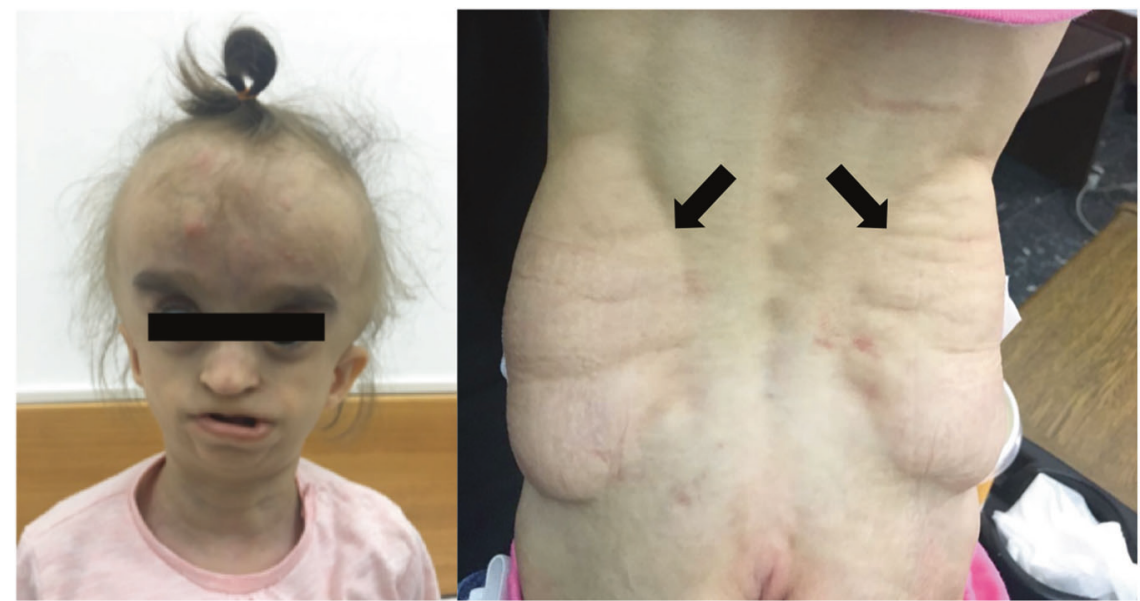

heterozygous variants of a splice site variant and a nonsense variant in the POLR3A gene in a female patient with WRS [8]. Moreover, 4H and WRS represent different clinical phenotype, with overlapping features such as hypomyelination, supporting the contention that biallelic variants within the POLR3A gene possibly associated to both syndromes. Lately, autosomal recessive adolescent-onset progressive spastic ataxia has also been speculated to be associated with biallelic variants within the POLR3A gene [10-12]. As most variations observed in the aforementioned syndromes are compound heterozygous, it is thought that the genotype-phenotype correlations probably depend on the level of functional POLR3A protein expressed in the cells, as well as additional genetic and epigenetic changes [4, 13].

Here we report a 5-year-old female that has classical clinical features of WRS and was shown to be compound heterozygous for variants in the POLR3A gene. Here we present a novel variation never reported before in the databases.

\section{Material and methods}

\section{Subject}

A 5-year-old female patient was admitted to Bursa Uludag University Hospital, Department of Medical Genetics with symptoms related to WRS. She was born at term to nonconsanguineous parents and the family history was negative for genetic syndromes (Supplementary Fig. 1). Written informed consent have been taken from her parents.

\section{Molecular genetic analyses}

The Illumina's commercial kit by Illumina TrueSight One panel was performed for targeted re-sequencing on an
Illumina MiSeq platform. An in-house pipeline was used to align the sequence reads with a reference genome, as well as variant calling, annotation and variant filtering to remove benign single nucleotide polymorphisms with allele frequency $\leq 0.03$ [14]. Public databases such as 1000 Genomes Project Database (http://www.internationalgenome.org/), Exome Variant Server (EVS) database (http://evs.gs.wa shington.edu/EVS/), Exome Aggregation Consortium (ExAC) database (exac.broadinstitute.org/), and the public database of single nucleotide variants (dbSNP, www.ncbi. nlm.nih.gov/SNP/) were used to filter and prioritize the variants and to check for allele frequencies, while the Human Gene Mutation Professional Database (HGMD) (http://www.biobase-international.com/product/hgmd) was used to identify genetic variations that underlie, or are closely associated with human inherited disease [15]. In silico prediction software such as SIFT (Sorting Intolerant From Tolerant), PolyPhen-2 (Polymorphism Phenotyping v2), and Mutation Taster, MutationAssessor and LRT (Likelihood Ratio Test) were used to assess the potential deleterious effects of missense variants [16-19]. Genetic variations were confirmed by PCR coupled with direct sequencing of target regions on a CEQ8800 Sequencer (Beckman Coulter) according to the manufacturer's protocols. Sanger sequencing, in search of any specific genetic variants found in the proband (IV.1), was also programmed for her parents.

\section{In silico analysis}

The homology modeling approach was used to generate the 3D structure of human RNA polymerase III subunit A (hPOLR3A) using the MODELLER software [20, 21]. Amino acid sequence corresponding to mRNA sequence of hPOLR3A (NM_007055.3) was retrieved from NCBI. SPPIDER server was used to determine the interacting residues of hPOLR3A (C160) with other subunits of RNA 
Fig. 2 Detected biallelic variants in the POLR3A gene. The patient inherited the POLR3A c. [3337-11T>C] intronic splice site variant from her mother and POLR3A c. $[3568 \mathrm{C}>\mathrm{T}]$ p.(Gln1190Ter) variant from her father $(\mathrm{P}$ proband, $\mathrm{F}$ father, $\mathrm{M}$ mother).

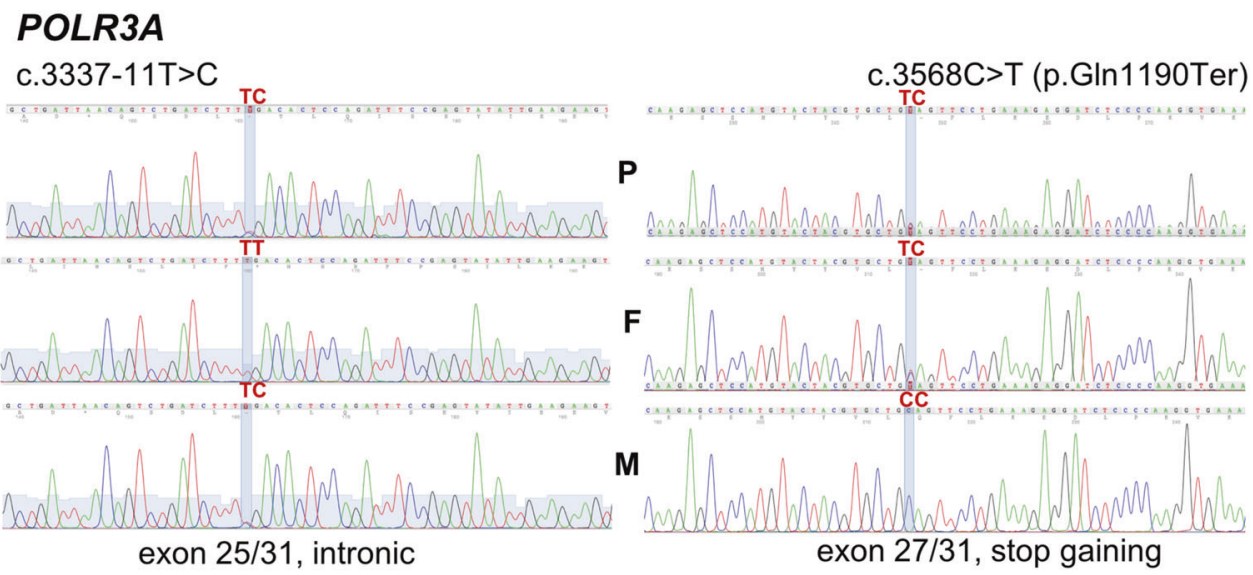

polymerase III. Molecular images were generated via PyMOL [22, 23].

\section{Results}

\section{Clinical examination}

Our patient was the first child of the family and was born 38th gestation week, with $2030 \mathrm{~g}$ birth weight from 26-yearold mother. During regular examination after eight days of birth her weight was $2185 \mathrm{~g}(<3 \mathrm{P})$, height was $45 \mathrm{~cm}(<3 \mathrm{P})$ and head circumference was $32 \mathrm{~cm}(<3 \mathrm{P})$. When she was a month and half, she was operated from her left upper eye-lid entropions. Her cardiological echocardiography examination have revealed asymmetric septal hypertrophy and patent foramen ovale. At the age of 9 months, nephrology examination has determined nephrolithiasis (left kidney with grade three dilations) and millimeter size kidney stones which were located in the left kidney. General clinical and dental examination indicated that she has local lipoatrophy, alopecia areata, osteopenia, natal teeth, and delayed dentition (Fig. 1) at the age of 6 years. Neurological examination showed developmental delay and electrophysiological (visual evoked potential) results showed bilateral message slowdown $(140,140)$ and bilateral ponto-mesencephalic message slowdown by MRI.

\section{Karyotyping and molecular genetics findings}

Cytogenetic analysis was normal 46, XX. The patient was then directed to MAGI Genetic and Rare Diseases Research, Diagnosis and Treatment Laboratory, Italy for molecular genetic analysis. Biallelic variants were detected in the POLR3A gene in the proband. She inherited the POLR3A c.3337-11T $>\mathrm{C}$ intronic splice site variant (ClinVar Submission ID: SUB7446850) from her mother and a novel POLR3A c.3568C >T, p.(Gln1190Ter) variant

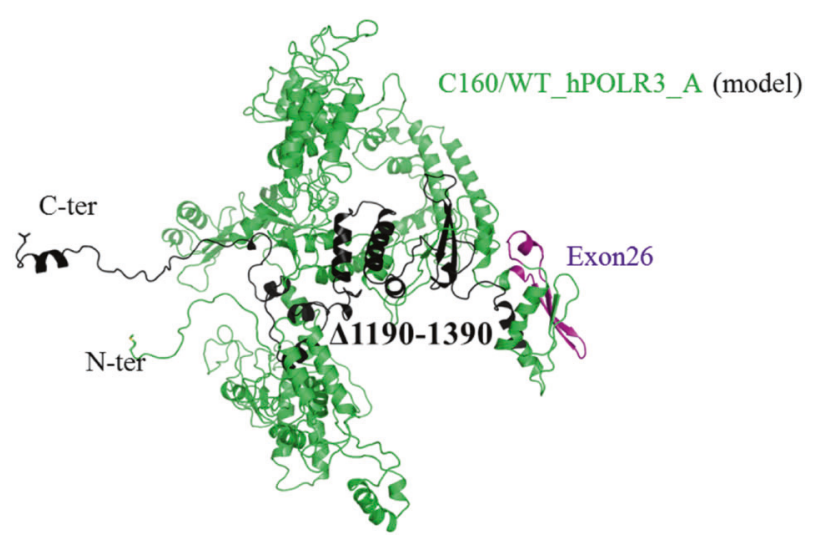

Fig. 3 Protein homology modeling of hPOLR3A. Protein sequence corresponding to NM_007055.3 mRNA sequence was used as the fulllength human RNA polymerase III subunit A (hPOLR3_A) sequence. NM_007055.3:c.3568C $>$ T introducing a stop codon after 1190th residue shown on the structure in black cartoon: $\Delta 1190-1390$. NG_029648.1(NM_007055.3):c.3337-11T>C causes skipping of exon 26. Position of exon 26 in C160 model is shown in magenta cartoon.

(ClinVar Submission ID: SUB714053) from her father (Fig. 2).

\section{In silico finding and functional predictions}

Sequence alignment of hPOLR3A via BlastP module of NCBI showed CryoEM structure of DNA-DIRECTED RNA POLYMERASE III SUBUNIT RPC1 from Saccharomyces cerevisiae, (PDB ID: 5fj8, chainA) has the highest sequence identity $(50.9 \%)$ among all other proteins having crystal structures. hPOLR3A was, therefore, modeled based on this structure (PDB: 5FJ8) (Fig. 3). Same procedure was performed to obtain the structure corresponding the NG_029648.1(NM_007055.3): c.3337$11 \mathrm{~T}>\mathrm{C}$ variation which causes hPOLR3A without exon 26: hPOLR3_A $\Delta 26$. Nucleotide and amino acid sequence information of POLR3A were retrieved from NCBI database with NM_007055.4 entry. Since sequences of each exon are 
Fig. 4 Model of POLR3A (C160, shown in green cartoon) and its interacting partners in RNA polymerase III elongation complex. Each protein in the complex is shown with surface representation and different colors. a Homology modeling analysis suggests that residues between 1190 and 1390 of POLR3A (inlet) interact with subunit $\mathrm{C} 128, \mathrm{C} 17$, and $\mathrm{C} 25$; residues translated from exon 26 (1112-1143) of POLR3A interact with $\mathrm{C} 11$ and $\mathrm{C} 128$. b Superimposing wild-type C160 (in green color) and C160 26 (in marine color) models suggest that exon skipping causes conformational changes in the region of $\mathrm{C} 11$ and C128 binding region of $\mathrm{C} 160$. Proteins interacting around the exon 26 of C160 is shown with surface representation.
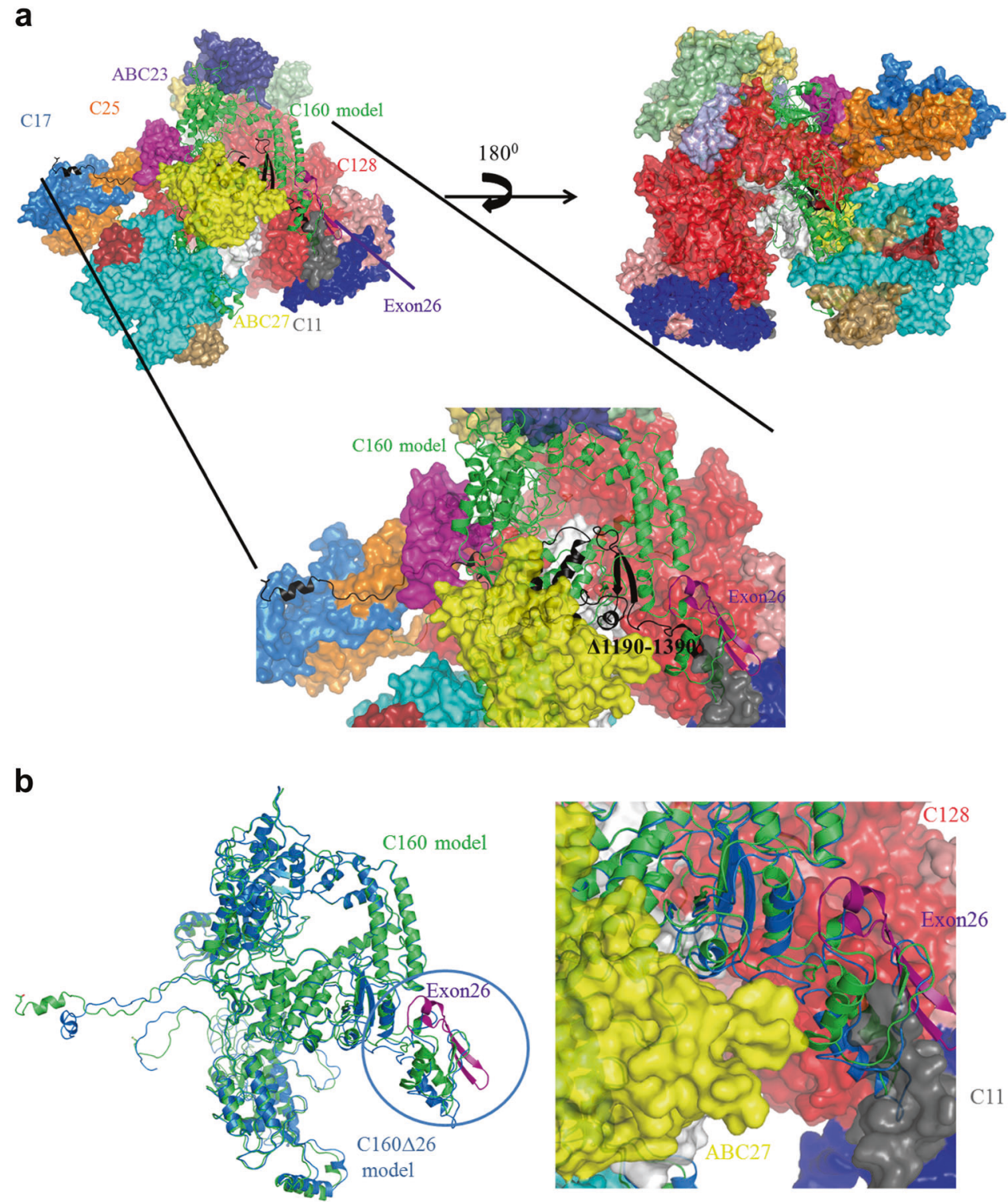

provided in the given NM entry sequentially, exon and amino acid numbers were determined manually according to data provided in the NCBI database under NM_007055.4 accession number.

Structure corresponding to NM_007055.3: c.3568C >T variation which introduces a stop codon was obtained by deleting residues after 1190th residues of full-length hPOLR3A (Fig. 3). Residues that participate in intersubunit contacts within the polymerase III model were mapped using SPPIDER.

\section{Discussion}

POLR3A encodes the largest subunit (C160) of the catalytic component of DNA-dependent RNA polymerase III, which synthesize small RNAs including tRNAs, 5S, and 7S rRNAs. In recent years, with the help of developing next generation sequencing techniques, variations in POLR3A was found to be associated with WRS. Patients were shown to carry biallelic variations on the POLR3A gene, each inherited from one parent, which results in the associated phenotype.

Some variations causing a diminished function of RNA polymerase III result in a deficiency of rRNA and/or tRNA, and this can cause persistent malfunction of cells and deregulated transcription of several ncRNAs [8]. Some of these ncRNAs, such as 7SL and 7SK RNAs, regulate the activity of DNA-dependent RNA polymerase II, hence the variants affecting POLR3A function can also affect levels of polymerase II-transcribed genes.

Despite the exact mechanisms through which a decrease in POLR3A leads to a clinical phenotype remains unclear, 
recent findings indicated that compound heterozygous variations in the POLR3A gene possibly result in the disease phenotype [4, 7-9]. POLR3A c.3568C $>$ T, p.(Gln1190Ter) variant was not previously detected in WRS patients. Likely, no reports are present in public databases regarding the effect of this variant on gene function. POLR3A c.3337$11 \mathrm{~T}>\mathrm{C}$ variant that was detected in a heterozygous state in our subject was previously detected in six individuals with WRS and was shown to cause skipping of exon 26 [24]. At the protein level, the splicing defect caused by the c.3337$11 \mathrm{~T}>\mathrm{C}$ variant results in an in-frame deletion represented as p.I1113_E1143del.

C160 subunit of the RNA polymerase III, which is encoded by POLR3A, plays a catalytic role [14]. Homology model of the complex shows that subunit $\mathrm{C} 128$ forms the active-center cleft of RNA polymerase III along with the C160 subunit. Other subunits e.g., $\mathrm{C} 17, \mathrm{ABC} 27, \mathrm{ABC} 23$, $\mathrm{C} 25$, and $\mathrm{C} 11$ are auxiliary subunits with specialized functions. hPOLR3A model and SPPIDER analysis indicates that totally 445 residues of $\mathrm{C} 160$ subunit is interacting with other subunits of the complex. Among those, 201 residues lie at the interface between the $\mathrm{C} 160$ subunit and any of the six interacting partners, namely the $\mathrm{C} 128, \mathrm{C} 11$, $\mathrm{C} 17, \mathrm{ABC} 27, \mathrm{ABC} 23$, and $\mathrm{C} 25$ of the elongation complex. The last 200 C-terminal amino acid residues (1190-1390) span the center of C160 which is sort of a hub to interact with different subunits of RNA polymerase III such as C128, C17, and C25. SPPIDER suggests that 65 of the interacting residues of $\mathrm{C} 160$ are found in 1190th and 1390th residues which are located at the $\mathrm{C}$-terminal of the protein (Fig. 4a). Since, these residues are completely missing in NM_007055.3:c.3568C $>$ T variant, the catalytic activity of RNA polymerase III is adversely affected. Although it is difficult to interpret the effect of exon skipping variations on the overall protein structure since it may change the folding pattern of the protein, we can simply say that nine of the interacting residues are in the amino acids expressed from exon 26. Homology model of hPOLR3A shows that amino acids corresponding to exon 26 interact with $\mathrm{C} 128$ and $\mathrm{C} 11$ (Fig. 4b). In the light of these findings we can speculate that absence of exon 26 may change the binding region of $\mathrm{C} 128$ and $\mathrm{C} 11$ on the $\mathrm{C} 160$ which may attenuate the overall activity of RNA polymerase III. These two variations on the C160 are predicted to have major effect on the role of RNA polymerase III by disrupting the subunit interactions.

It was shown that mutated polymerase III results in an insufficient number of mature ribosomes in the neuroepithelium and neural crest cells at a critical time point during embryogenesis [25]. Complete loss of function is quite likely to be incompatible with life. The splice-variant present in our patient may allow some expression of the protein and perhaps amelioration of a lethal phenotype, which allowed this patient's survival.
Previous studies indicated that the variant, c.3337$11 \mathrm{~T}>\mathrm{C}$, was represented in WRS patients with its cis POLR3A c.[3337-11T>C; $1909+22 \mathrm{G}>\mathrm{A}]$. However, recently Wambach et al. reported that the c.3337-11T $>C$ variant gives WRS phenotype with other biallelic, rare variants in compound heterozygous manner [4].

Overall, our study gives strong evidential support that POLR3A c.3337-11T $>\mathrm{C}$ variant itself, without its cis POLR3A [c.3337-11T >C; c. $1909+22 \mathrm{G}>\mathrm{A}$ ], is enough to show the WRS disease phenotype in compound heterozygous manner POLR3A [c.3568C $>\mathrm{T}]$; [c.3337-11T $>\mathrm{C}]$ variant. However, this study is in agreement with previous findings that severe loss of function due to biallelic POLR3A variations is associated with WRS. Further analysis of the ribosomal biogenesis pathway will identify other conditions belonging to the ribosomopathy phenotype and will possibly provide new molecular targets for diseasespecific drug development.

\section{Compliance with ethical standards}

Conflict of interest The authors declare that they have no conflict of interest.

Publisher's note Springer Nature remains neutral with regard to jurisdictional claims in published maps and institutional affiliations.

\section{References}

1. Arboleda G, Morales LC, Quintero L, Arboleda H. Neonatal progeroid syndrome (Wiedemann-Rautenstrauch syndrome): report of three affected sibs. Am J Med Genet A. 2011;155a:1712-5.

2. Arboleda G, Ramirez N, Arboleda H. The neonatal progeroid syndrome (Wiedemann-Rautenstrauch): a model for the study of human aging?. Exp Gerontol. 2007;42:939-43.

3. Akawi N, Ali B, Al Gazali L. A progeroid syndrome with neonatal presentation and long survival maps to $19 \mathrm{p} 13.3 \mathrm{p} 13.2$. Birth Defects Res A Clin Mol Teratol. 2013;97:456-62.

4. Wambach JA, Wegner DJ, Patni N, Kircher M, Willing MC, Baldridge $\mathrm{D}$, et al. Bi-allelic POLR3A loss-of-function variants cause autosomal-recessive Wiedemann-Rautenstrauch syndrome. Am J Hum Genet. 2018;103:968-75.

5. Bernard G, Vanderver A. POLR3-Related Leukodystrophy. 2012 Aug 2 [Updated 2017 May 11]. In: Adam MP, Ardinger HH, Pagon RA, et al., editors. GeneReviews ${ }^{\circledR}$ [Internet]. Seattle (WA): University of Washington, Seattle; 1993-2020. Available from: https://www.ncbi.nlm.nih.gov/books/NBK99167.

6. Bernard G, Chouery E, Putorti ML, Tetreault M, Takanohashi A, Carosso G, et al. Mutations of POLR3A encoding a catalytic subunit of RNA polymerase Pol III cause a recessive hypomyelinating leukodystrophy. Am J Hum Genet. 2011;89:415-23.

7. Paolacci S, Bertola D, Franco J, Mohammed S, Tartaglia M, Wollnik B, et al. Wiedemann-Rautenstrauch syndrome: a phenotype analysis. Am J Med Genet A. 2017;173:1763-72.

8. Jay AM, Conway RL, Thiffault I, Saunders C, Farrow E, Adams $\mathrm{J}$, et al. Neonatal progeriod syndrome associated with biallelic truncating variants in POLR3A. Am J Med Genet A. 2016; 170:3343-6. 
9. Lessel D, Ozel AB, Campbell SE, Saadi A, Arlt MF, McSweeney KM, et al. Analyses of LMNA-negative juvenile progeroid cases confirms biallelic POLR3A mutations in WiedemannRautenstrauch-like syndrome and expands the phenotypic spectrum of PYCR1 mutations. Hum Genet. 2018;137:921-39.

10. Minnerop M, Kurzwelly D, Wagner H, Soehn AS, Reichbauer J, Tao F, et al. Hypomorphic mutations in POLR3A are a frequent cause of sporadic and recessive spastic ataxia. Brain. 2017;140:1561-78.

11. Gauquelin L, Tetreault M, Thiffault I, Farrow E, Miller N, Yoo B, et al. POLR3A variants in hereditary spastic paraplegia and ataxia. Brain. 2018;141:e1.

12. Minnerop M, Kurzwelly D, Rattay TW, Timmann D, Hengel H, Synofzik M, et al. Reply: POLR3A variants in hereditary spastic paraplegia and ataxia. Brain. 2018;141:e2.

13. Báez-Becerra CT, Valencia-Rincón E, Velásquez-Méndez K, Ramírez-Suárez N, Guevara C, Sandoval-Hernandez A, et al. Nucleolar disruption, activation of P53 and premature senescence in POLR3A-mutated Wiedemann-Rautenstrauch syndrome fibroblasts. BioRxiv. 2020. https://www.biorxiv.org/content/10. 1101/2020.01.29.925131v1.full.

14. Marceddu G, Dallavilla T, Guerri G, Manara E, Chiurazzi P, Bertelli M, et al. PipeMAGI: an integrated and validated workflow for analysis of NGS data for clinical diagnostics. Eur Rev Med Pharmacol Sci. 2019;23:6753-65.

15. Stenson PD, Mort M, Ball EV, Evans K, Hayden M, Heywood S, et al. The Human Gene Mutation Database: towards a comprehensive repository of inherited mutation data for medical research, genetic diagnosis and next-generation sequencing studies. Hum Genet. 2017;136:665-77.
16. Sim NL, Kumar P, Hu J, Henikoff S, Schneider G, Ng PC. SIFT web server: predicting effects of amino acid substitutions on proteins. Nucleic Acids Res. 2012;40:W452-7.

17. Adzhubei IA, Schmidt S, Peshkin L, Ramensky VE, Gerasimova A, Bork P, et al. A method and server for predicting damaging missense mutations. Nat Methods. 2010;7:248-9.

18. Schwarz JM, Cooper DN, Schuelke M, Seelow D. MutationTaster2: mutation prediction for the deep-sequencing age. Nat Methods. 2014;11:361-2.

19. Reva B, Antipin Y, Sander C. Predicting the functional impact of protein mutations: application to cancer genomics. Nucleic Acids Res. 2011;39:e118.

20. Webb B, Sali A. Comparative protein structure modeling using MODELLER. Curr Protoc Bioinform. 2016;54:5.6.1-37.

21. Marti-Renom MA, Stuart AC, Fiser A, Sanchez R, Melo F, Sali A. Comparative protein structure modeling of genes and genomes. Annu Rev Biophys Biomol Struct. 2000;29:291-325.

22. Ding Z, Kihara D. Computational methods for predicting proteinprotein interactions using various protein features. Curr Protoc Protein Sci. 2018;93:e62.

23. DeLano WL. The PyMOL molecular graphics system. 2008. http://pymol.org.

24. Paolacci S, Li Y, Agolini E, Bellacchio E, Arboleda-Bustos CE, Carrero D, et al. Specific combinations of biallelic POLR3A variants cause Wiedemann-Rautenstrauch syndrome. J Med Genet. 2018;55:837-46.

25. Dauwerse JG, Dixon J, Seland S, Ruivenkamp CA, van Haeringen A, Hoefsloot LH, et al. Mutations in genes encoding subunits of RNA polymerases I and III cause Treacher Collins syndrome. Nat Genet. 2011;43:20-2. 